\title{
Content-Based Image Retrieval Using Modified Human Colour Perception Histogram
}

\author{
Shaila S.G and A.Vadivel \\ Department of Computer Applications, National Institute of Technology, \\ Tiruchirappalli 620 015, India, Fax: +91-431-2500133 \\ shaila@nitt.edu vadienitt.edu
}

\begin{abstract}
This paper proposes Modified Human Colour Perception (MHCPH) based on human visual perception. The colour and gray weights are distributed to neighbouring bins smoothly with respect to pixel information. The amount of weight distributed to the neighbouring bins is estimated using NBS distance, which is for human visual perception of colour. This distribution makes it possible to extract the background colour information effectively along with the foreground information. The low-level feature of all the database images are extracted and stored in feature database. The relevant images are retrieved for a query based on the similarity ranking between the query and database images. In this work, Manhattan distance is used as distance metric. The experimental results are promising and show that the proposed approach identifies relevant images based on the level of smooth distribution even for an image with complex background colour.
\end{abstract}

\section{Keywords}

Content based image retrieval, Human colour perception, HSV colour space, Smooth distribution, MHCPH histogram, NBS distance.

\section{Introduction}

Various techniques have been proposed in academia and industry for image retrieval applications. These approaches can roughly be classified into three categories such as text-based retrieval, content-based retrieval and semantic-based retrieval. In the text-based retrieval technique, each image has a number of keywords for describing the image and the keyword based matching is performed to retrieve relevant images. In content-based image retrieval applications, various well-known low-level features like colour, texture and shape are extracted for describing the image semantics. In the semantic-based retrieval technique, semantic is used to retrieve relevant images. In recent years, attention is focused by researchers on Content-Based Image Retrieval (CBIR), which is a sub-problem of content-based retrieval. It is noticed that the size of the image database used in image retrieval is increasing exponentially and hence it is necessary to propose and use effective tools for retrieving images. Well-known and most popular image retrieval systems are QBIC[13], NeTra[11], PicToSeek[6], Blobworld[1], etc.

Natarajan Meghanathan, et al. (Eds): ITCS, SIP, JSE-2012, CS \& IT 04, pp. 229-237, 2012. 
In this paper, our goal is to retrieve set of images that are similar to a given query image. In image retrieval applications, it has been observed that the colour histogram based approach is well suited, since colour matching generates the strongest perception of similarity to the human eye. It is often represented in the form of a histogram, which is a first-order statistical measure that captures the global distribution of colour in a given image. This can be represented in various forms such as colour histogram [15], colour moments and cumulative colour histogram [16]. A colour histogram may be generated in the RGB colour space, HSV colour space, YCbCr colour space, etc. Since, the RGB colour space is having some drawbacks such as, it does not explicitly distinguish between colour and intensity components, other colour space like $\mathrm{HSV}$, YCbCr, which separate saturation and intensity components are frequently used. RGB values by suitable conversions may be computed to get the values for each component in HSV colour space. Some of the recently proposed colour representatives are colour saliency histogram [23], which supports human visual attention principle and based on the features of colour, orientation and intensity and followed by the difference of Gaussians and normalization processing, the comprehensive saliency map of an image is generated. Recently, circular ring histogram[22] has been proposed, which has the spatial information. The image is segmented initially using group of circular rings and then the histogram is constructed using the segmented rings. The statistical feature of image blocks has been extracted for representing the colour of blocks, which is named as Order Based Block Colour Feature[19].In this approach image is divided into 48 blocks and the feature is extracted. In our proposed work, we modify the HCPH histogram [21] to capture the colour information in high-dense background of images for retrieval applications. The weight calculated is distributed to the neighbouring bins based on NBS distance and the performance of the modified $\mathrm{HCPH}$ is encouraging while retrieving high-dense background images.

The outline of this paper is as follows. Section 2 presents the related works and proposed technique is explained in Section 3. In Section 4, the experimental results are presented and we conclude the paper in the last section

\section{Related Works}

In colour-based image retrieval, there are primarily two methods: one based on colour layout[18] and the other based on colour histogram[3]. In the colour layout approach, two images are matched by their exact colour distribution. This means that two images are considered close if they not only have similar colour content, but also if they have similar colour in approximately the same positions. In the second approach, each image is represented by its colour histogram. A histogram is a vector, whose components represent a count of the number of pixels having similar colours in the image. Thus, a colour histogram may be considered to be a signature extracted from a complete image. Colour histograms extracted from different images are indexed and stored in a database. During retrieval, the histogram of a query image is compared with the histogram of each database image using a standard distance metric like Euclidean distance or Manhattan distance. Since, colour histogram is a global feature of an image, the approaches based on colour histogram are invariant to translation, rotation and scale.

Various techniques has been proposed to represent the colour of an image [2][7][9][10][14][17]. It is found that robust histogram construction scheme using the HSV colour space in which a perceptually smooth transition is captured based on the Human Visual Perception of colour $(\mathrm{HCPH})$ can represent semantic information up to a certain degree, due to the complexity in background [21]. In colour saliency histogram [24], extraction of salient regions is based on the bottom-up visual attention model. Although it has introduced prior knowledge in the model, the bottom-up attention is only suitable for the primary stage of visual perception, it has limitations. Order-based Block Colour Feature [19] is one type of image's colour feature. It has an advantage of the local colour statistical information but it has some bionic traits that this colour feature 
cannot alone suffice for CBIR. It has to be combined with other features such as shape, texture, and so on. Hence, Modified HCPH (MHCPH), which is an improved version of HCPH is developed. In $\mathrm{HCPH}$, each pixel is divided into true colour component and grey colour component, in a single iteration. But in $\mathrm{MHCPH}$, we distribute the true colour components and grey colour components into many levels thus distributing the weights to neighbouring bins based depending on the weight values and NBS distance.

\section{Modified HCPH}

Pixels in a image can be represented with the combination of Hue, Saturation and Intensity Value in the HSV colour space. It is a three dimensional hexacone representation and the central vertical axis is intensity, I. Hue is an angle in the range $[0,2 \pi]$ and is relative to the red axis with red at angle 0 , green at $2 \pi / 3$, blue at $4 \pi / 3$ and red again at $2 \pi[23]$ respectively. Saturation, $S$, is measured as a radial distance from the central axis with value between 0 at the centre to 1 at the outer surface. This is represented in Fig.1.

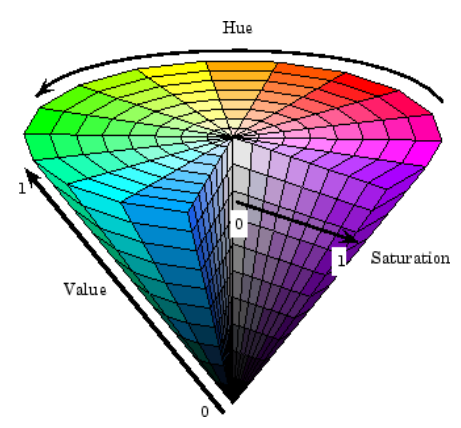

Fig 1. HSV Colour Model

The pixel colour is approximated by its intensity or by its hue, say for example while the intensity is low and saturation is high, a pixel colour is very much close to the grey colour. Similarly for other combination of intensity and saturation, we can approximate the pixel value the other way. Therefore, the saturation and intensity values of a pixel are used to determine whether it can be treated as a true colour pixel or a gray colour pixel. It is emphasized that this approach treats the pixels as a distribution of "colours" in an image where a pixel may be of a "gray colour" (i.e., somewhere between black and white, both inclusive) or of a "true colour" (i.e., somewhere in the red, green, blue, red spectrum). The reason is that, for an observer, this is what an image represents is a collection of points having colours - red, yellow, green, blue, black, gray, white, etc.

In our proposed work, colour information of each pixel is converted to HSV colour space. An image pixel contains true colour components, in which the dominant factor is hue. Intensity is the dominant factor for grey colour components. In the first iteration, we calculate true colour components and grey colour components and they are smoothly (i.e., iteratively) distributed, based on hue and intensity values of neighbouring pixel. This is shown in Fig.2. 


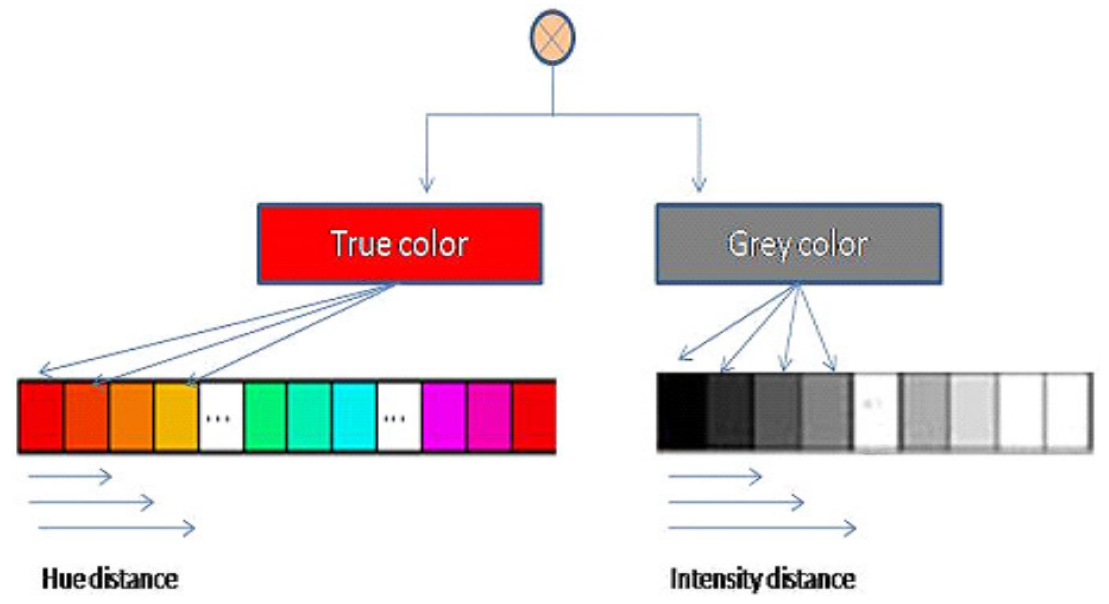

Fig 2. Distribution of true colour and grey colour components

As noticed form Fig. 2, the weights are distributed to the neighbouring bins. The colour information obtained in first level is taken as reference and is distributed smoothly into more iteration. In this paper, we have considered five iteration levels. NBS distance [23] is calculated using true colour and grey colour for distributing the pixel weight to the neighbouring bins. The distance between true colour weight of reference pixel and its iterative distribution with adjacent bins is calculated. Since, soft decision is done for a single pixel, saturation appears to be very small and hence $\Delta \mathrm{S}$ is considered as 0 . The $\Delta \mathrm{I}$ is considered as the distance calculation and is only for true colour which is shown in Eq. (1).

$$
d(\vec{x}, \vec{y})=1.2^{*} \sqrt{2 x_{2} y_{2}\left(1-\cos \left(\frac{2 \pi \Delta H}{100}\right)\right)+\Delta S^{2}+(4 \Delta I)^{2}}
$$

In Table.1, we present the NBS distance value, which gives the colour difference with respect to the human visual perception based on the distance range. Considering the colour difference and distance, the weight of true colour is distributed. Since, true colour is smoothly distributed and true colour weight also should be smoothly distributed. As per Eq. (1), for true colour, $\Delta \mathrm{S}$ and $\Delta \mathrm{I}$ remains zero. This is due to the fact that the saturation and intensity distributed difference is minimum with respect to a single pixel.

Table 1. NBS distance Table

\begin{tabular}{|l|l|}
\hline NBS Value & Human Perception \\
\hline $0 \sim 1.5$ & Almost the same \\
\hline $1.5 \sim 3.0$ & Slightly different \\
\hline $3.0 \sim 6.0$ & Remarkably different \\
\hline $6.0 \sim 12.0$ & Very different \\
\hline $12.0 \sim$ & Different colour \\
\hline
\end{tabular}


For grey colour, $\Delta \mathrm{S}$ and $\Delta \mathrm{H}$ remains zero, since saturation and hue difference appears to be very minimum. Based on these conditions, true colour weight and grey colour weights are distributed. NBS distance is calculated to the adjacent bins of true colour, it is observed that the immediate adjacent bin close to the reference bin will lie in the distance ranging from $0 \sim 1.5$. As per the NBS table it is "almost the same". Thus $100 \%$ of true colour weight will be distributed to the immediate adjacent bin. As smooth distribution of hue goes on, the NBS distance is calculated for all the adjacent bins iteratively. We observe that while distance starts increasing, say in the range of 1.6 3.0, there is a "slight colour difference". We divide it into 3 groups with 15 iterations. Now, the weight is distributed from 99 to 85 . When the distance is measured with respect to the adjacent bins in the range of 3.1 6.0, there is a "remarkable colour difference" range. Hence, we have divided it into 6 groups with 30 iterations and weight distribution starts from 84 to 55 . As distance increases, the colour difference will be more, which may lie in the range of 6.1 12.0 and is represented as "very different colour". We divide it into 12 groups with 60 iterations and weight distribution starts from 54 to 00 , if distance is greater than 12.0 , then it is totally different colour and weight distribution is $0 \%$.

The percentage of weight distribution to the adjacent bins is calculated using Eq. (2) and the same is represented in the form of tree in Fig.3.

$$
S W_{H(S, I)}=100-[(N B S-1.5) * 10]
$$

Where NBS - True colour distance

During grey colour weight distribution, intensity is distributed iteratively or smoothly. However, we need not apply NBS distance, since the colour difference is very minimum. Thus, we distribute our grey colour weight using Eq. (3).

$$
S W_{I(S, I)}=1-S W_{H(S, I)}
$$

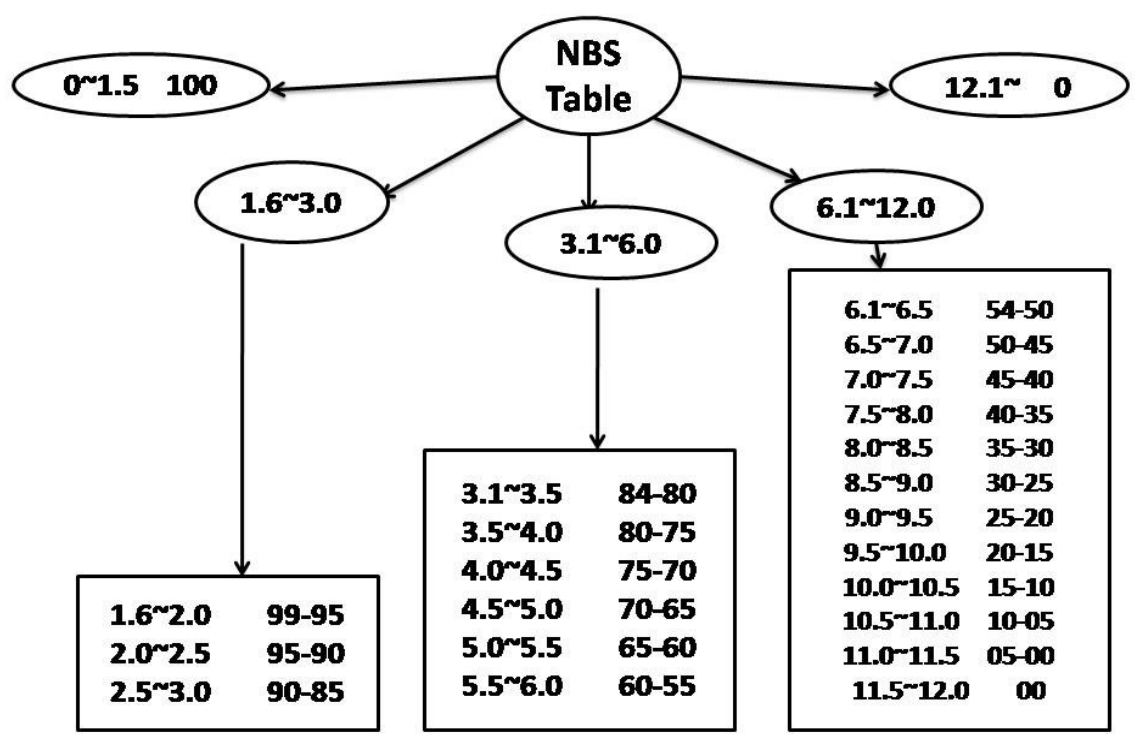

Fig 3. Smooth distribution of true colour weight 


\section{Experimental Results}

In CBIR application, Precision and Recall are used as parameters for evaluating the performance. Precision is the ratio of the number of the relevant images retrieved to the total number of the irrelevant and relevant images retrieved. Precision is defined as:

$$
\text { Precision }=\frac{R_{r}}{T_{r}}
$$

Where $R_{r}$ is number of relevant images retrieved. $T_{r}$ is the total images retrieved.

Recall is defined as the ratio of the number of relevant images retrieved to the total number of the relevant images in the database. Recall is a measure of completeness.

$$
\text { Recall }=\frac{R_{r}}{T}
$$

Where $R_{r}$ is number of relevant images retrieved. $T$ is the total relevant images retrieved.

In order to evaluate the performance of the proposed scheme, coral benchmark database images are used, which consists of 10 classes such as people, vehicle, building, flower, horses, etc. In experiments, the HSV colour space is chosen for chromatic image processing. We have selected large number of query images from different categories and retrieved the result for each query. Average Precision and Recall is calculated and is shown in the Fig.4. From the figure ,It is observed that for lower values of recall, the precision is getting higher, which is greater than $65 \%$. Similarly, for higher value of recall, the precision is comparable and the performance of the proposed method is encouraging.

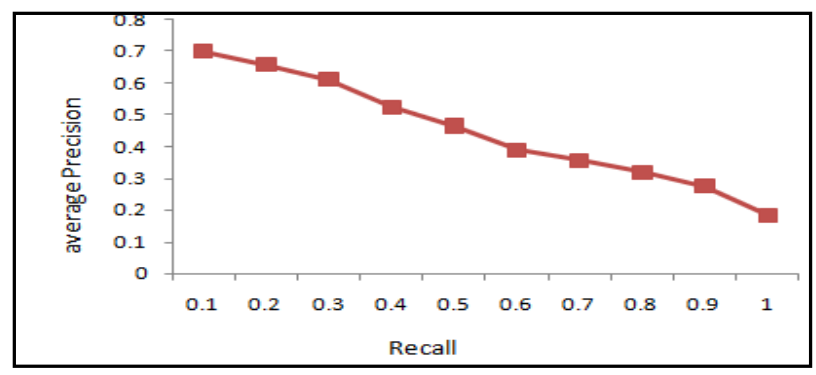

Fig 4. Average Precision versus Recall

In addition, we have compared the performance of the proposed method with DCT histogram quantization algorithm [12] and are shown in Table.2. In the DCT Histogram Quantization, due to the complex background with respect to a object, the efficiency in the retrieval rate of relevant images has gone down to $40 \%$ in the sample dataset of elephant. However, for Dinosaurs category, the precision of has increased to $99 \%$. Here, the retrieval rate is highly dependent on the content of background. However, the precision rate is not completely influenced by the background content of the images on the proposed approach. 
Table 2. Performance comparison between DCT Quantization and MHCPH

\begin{tabular}{|l|c|c|}
\hline \multirow{2}{*}{ Class } & \multicolumn{2}{|c|}{ Average precision } \\
\cline { 2 - 3 } & MHCPH & DCT \\
\hline Dinosaur & 0.86 & 0.99 \\
\hline Elephant & 0.60 & 0.40 \\
\hline
\end{tabular}

Since, weight is smoothly distributed to neighbouring bins based on the true colour and gray colour weight, the entire information of the background as well as the object is captured and thus the precision retrieval is 60\%. Fig.5 and Fig.6 depicts sample retrieval result for both the proposed and DCT based histogram. The image on the top centre is the query and the rest of the image below is the retrieved image set.

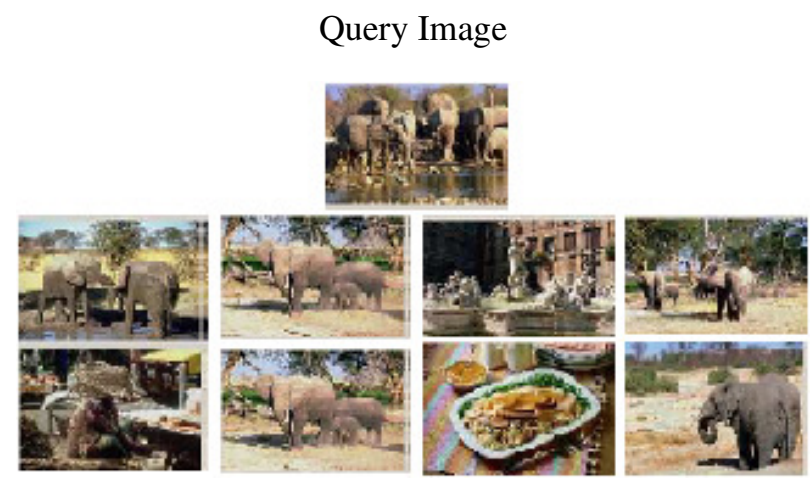

Fig 5. Sample Retrieval set using DCT Quantization.

Query Image

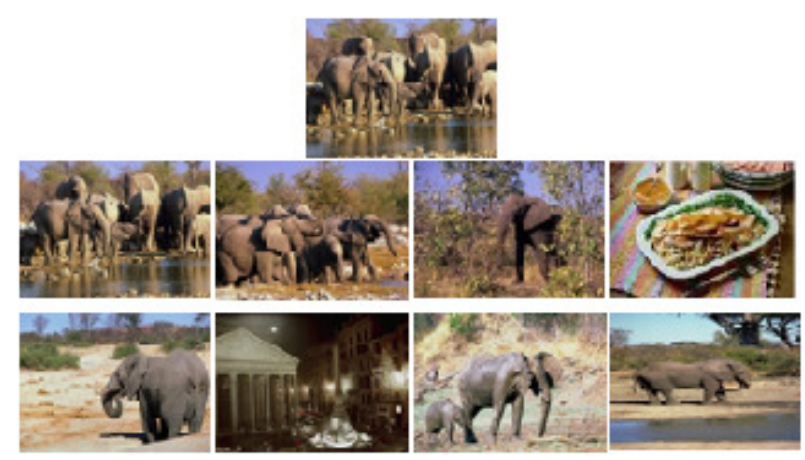

Fig 6. Sample Retrieval set using $\mathrm{MHCPH}$

\section{Conclusion}

In this paper, we have proposed content based image retrieval technique using $\mathrm{MHCPH}$. By extracting the colour features of each pixel of the image, the smooth distribution is done with 
respect to true colour and grey colour. Manhattan distance is used to measure the similarity distance in order to retrieve the similar images from the database. In our work, we took only colour feature and smoothly distributed its information for effective retrieval. However, further increase in the iterations, will certainly improve the retrieval results with high precision and recall. Thus, further care will be taken to balance both of these parameters. For future work, we plan to improve the rate of retrieval by adding more iterations and adding extra features such as texture and shape with the colour to improve the relevance.

\section{Acknowledgment}

The work done by Dr. A.Vadivel and is supported by research grant from the Department of Science and Technology, India, under Grant DST/TSG/ICT/2009/27 dated 3rd September 2010.

\section{References}

[1] Carson, C., Thomas, M., Belongie, S., Hellerstein, JM, \& Malik, J, (1999) "Blobworld: A system for region-based image indexing and retrieval", Third Int. Conf. on Visual Information Systems, pp 217225.

[2] Deng, Y., Manjunath, B.S., Kenney, C., Moore, M.S. \& Shin, H. (2001) "An efficient colour representation for image retrieval”, IEEE Transactions on Image Processing, Vol. 10, pp140-147.

[3] Deb \& Y. Zhang, (2004) “An overview of content-based Image retrieval techniques," Proc. on 18th Int. Conf. on Advanced Information Networking and Applications, Vol. 1, pp59-64.

[4] Fuxiang Lu, Xiaokang Yang, Rui Zhang, \& Songyu Yu , (2009) "Image classification based on pyramid histogram of topics," Multimedia and Expo, ICME 2009. IEEE International Conference on , pp398-401.

[5] Gevers, T. \& Smeulders, A.W.M., (1999) "Colour-based object recognition", Pattern Recognition, Vol. 32, pp453-464.

[6] Gevers,T \& Smeulders, A.W.M., (2000) "PicToSeek: Combining colour and shape invariant features for image retrieval”, IEEE Trans. on Image Processing, Vol. 9 pp102-119.

[7] Han, J. \& Ma, K-K., (2002) "Fuzzy colour histogram and its use in colour image retrieval", IEEE Transactions on Image Processing, Vol. 2, pp944-952.

[8] Jain, A \& Vailaya,A ,(1996) " Image retrieval using colour and shape”, Pattern Recognition, Vol. 29 pp1233-1244.

[9] Kender, J.R., (1976) "Saturation, Hue and Normalised Colour: Calculation, Digitisation and Use", Computer Science Technical Report, Carnegie-Mellon University, Pittsburg, USA.

[10] Lei, Z., Fuzong, L. \& Bo, Z., (1999) “A CBIR method based colour-spatial feature”, Proc. IEEE Region 10 Annual International Conference on TENCON 99, Proceedings ,Cheju Island, South Korea, pp166-169.

[11] Ma W.Y. \& Manjunath, B.S., (1997) "NeTra: A toolbox for navigating large image databases", IEEE Int. Conf. on Image Processing pp568-571.

[12] Mohamed, A., Khellfi, F., Ying Weng, Jianmin Jiang \& Ipson, S., (2009) "An efficient Image Retrieval through DCT Histogram Quantization" , International Conference on CyberWorlds,, pp237-240.

[13] Niblack,W, Barber,R Equitz,W, Flickner,M, Glasman,E,.Petkovic,D Yanker,P,.Faloutsos,C \& Taubin,G., (1993) "The QBIC project: Querying images by content using colour, texture and shape", SPIE Int. Soc. Opt. Eng., in Storage and Retrieval for Image and Video Databases.Vol. 1908, pp173187. 
[14] Nezamabadi-pour, H. \& Kabir, E., (2004) "Image retrieval using histograms of uni-colour and bicolour blocks and directional changes in intensity gradient”, Pattern Recognition Letters, Vol. 25, pp1547-1557.

[15] Swain, M.J \& allard, D.H., (1991) “Colour Indexing”, Computer Vision. Vol. 7, pp11-32.

[16] Stricker, M A. \& Orengo, M., (1995) “Similarity of colour images”, SPIE, Vol. 2420, pp381-392.

[17] Shih, J.L. \& Chen, L.H., (2002) "Colour image retrieval based on primitives of colour moments", Proc. IEEE Vision, Image and Signal Processing, pp88-94.

[18] Smith, J. R., \& Chang, S.-F., (1996) "VisualSEEk: A Fully Automated Content-Based Image Query System", ACM Multimedia, pp87-98

[19] Shoujue Wang \& Hong Qin , (2009) "A Study of Order-Based Block Colour Feature Image Retrieval Compared with Cumulative Colour Histogram Method", Sixth International Conference on Fuzzy Systems and Knowledge Discovery, Vol. 1, pp81-84.

[20] Vadivel A. ,Majumdar A. K. \& Sural Shamik, (2003) "Perceptually Smooth Histogram Generation from the HSV Colour Space for Content Based Image Retrieval", International Conference on Advances in Pattern Recognition, Kolkata, pp 248-251.

[21] Vadivel.A, Shamik Sural \& A.K.Majumdar, (2008) "Robust Histogram Generation from the HSV Space based on Visual Colour Perception", International Journal of Signal and Imaging Systems Engineering, InderScience, Vol. 1(3/4), pp245-254.

[22] Wang \& Xiaoling, (2009) "A Novel Circular Ring Histogram for Content-Based Image Retrieval", First International Workshop on Education Technology and Computer Science, Vol. 2, pp785-788.

[23] Yihong Gong, Proietti, G \&, Faloutsos, C., (1998) "Image indexing and retrieval based on human perceptual color clustering,". Proc. IEEE Computer Society Conference on Computer Vision and Pattern Recognition, pp.578-583.

[24] Yunqi Lei, Zhenxiang Shi, Xutuan Jiang, Qingmin Li \& Dongjie Chen , (2009) "Image Retrieval Based on Colour Saliency Histogram", International Symposium on Computer Network and Multimedia Technology, pp1-4.

\section{Authors}

\section{Short Biography}

Shaila S.G received her M-Tech in Computer Science and Engineering in 2005 from M.V.Jayaraman College of Engineering, Vishweshwarayya Technological University, Bangalore, India. Currently she is a research scholar in the Department of Computer Applications, National Institute of Technology, Tiruchirappalli, India. Her research interest is Multimedia Information retrieval from distributed environment.

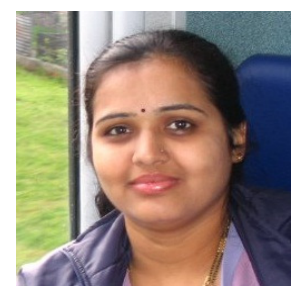

A. Vadivel is an Associate Professor at the National Institute of Technology, Tiruchirappalli, India. He received his MTech and $\mathrm{PhD}$ from the Indian Institute of Technology (IIT), Kharagpur, India, in 2000 and 2006 respectively. His research interest includes image and video processing, medical image analysis, object tracking, multimedia information retrieval from web and frequent pattern mining. He was awarded an Indo-US Research Fellow Award in 2008 by the Indo-US Science and Technology Forum, India and young scientist award by

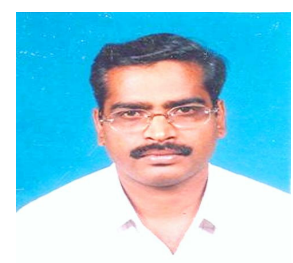
DST Government of India in 2007. 